\title{
Outcomes of patients in the national cancer database treated non-surgically for localized rectal cancer
}

\author{
Emma B. Holliday ${ }^{1}$, Pamela K. Allen ${ }^{2}$, Hesham Elhalawani ${ }^{1}$, Omar Abdel-Rahman ${ }^{3}$ \\ ${ }^{1}$ Department of Radiation Oncology, ${ }^{2}$ Department of Biostatistics, The University of Texas MD Anderson Cancer Center, Houston, Texas, USA; \\ ${ }^{3}$ Clinical Oncology Department, Faculty of Medicine, Ain shams University, Cairo, Egypt \\ Contributions: (I) Conception and design: EB Holliday, O Abdel-Rahman; (II) Administrative support: None; (III) Provision of study materials or \\ patients: None; (IV) Collection and assembly of data: H Elhalawani; (V) Data analysis and interpretation: PK Allen; (VI) Manuscript writing: All \\ authors; (VII) Final approval of manuscript: All authors. \\ Correspondence to: Emma B. Holliday, MD. Division of Radiation Oncology, The University of Texas MD Anderson Cancer Center, 1515 Holcombe \\ Blvd Unit 97, Houston, Texas 77030, USA. Email: ebholliday@mdanderson.org.
}

Background: Some patients undergo a non-operative approach to localized rectal adenocarcinoma either because they decline surgery or because their medical comorbidities preclude surgical intervention. Published studies reporting excellent outcomes with a "wait-and-see" approach have been small and highlyselected. We aimed to analyze survival outcomes and prognostic factors for patients with localized rectal adenocarcinoma in the National Cancer Database (NCDB) undergoing definitive radiation without surgical intervention.

Methods: The NCDB was queried for patients with non-metastatic rectal adenocarcinoma treated with definitive radiotherapy who did not undergo a surgical resection either because the patient refused surgery, surgery was medically contraindicated, or surgery was otherwise unplanned. Patient, tumor and treatmentrelated characteristics were compared between those treated with 45-50.3 Gray (Gy), 50.4-54 Gy and >54 Gy. Survivals were compared using the Log-Rank test. Univariate and multivariate Cox regression analyses were performed. Survivals were then compared utilizing a robust inverse-probability-weighted regression adjustment method with nearest-neighbor matching.

Results: Eight thousand four hundred and eight patients were included for analysis. After case-matching and adjusting for significant prognostic factors, patients receiving 50.4-54 Gy had a significantly longer median, 1- and 5-year overall survival (OS) (49.4 months, 85.8\%, 44.7\%) compared with patients receiving $45-50.3$ or $>54$ Gy (37.2 months, $79.2 \%, 38.4 \%$ and 34.2 months, $84.5 \%$, 35.3\%, respectively; Log rank P value $<0.0001)$.

Conclusions: In an unselected group of patients treated at NCDB-participating institutions, survival rates with a non-surgical approach to non-metastatic rectal adenocarcinoma are much lower than those reported in well-selected single-institutional studies. Moderate dose escalation from 50.4-54 Gy was associated with better OS compared with doses $<50.4$ Gy or >54 Gy after adjusting for significant covariant.

Keywords: Rectal cancer; chemotherapy; radiotherapy; non-surgical treatment

Submitted Jan 25, 2018. Accepted for publication Mar 01, 2018.

doi: 10.21037/jgo.2018.03.06

View this article at: http://dx.doi.org/10.21037/jgo.2018.03.06 


\section{Introduction}

In spite of the advances in screening, diagnosis and treatment strategies; colorectal cancer remains the third most commonly diagnosed cancer in the United States as well as an important cause of suffering for many patients worldwide $(1,2)$. Although survival rates for rectal adenocarcinoma are high in the modern era, its treatment brings about special challenges given the effects curative surgery and adjunctive therapies on sphincter function and quality of life $(3,4)$.

The treatment strategies for rectal cancer evolved significantly over the past three decades. Initially, radical surgery was considered the only line of treatment for non-metastatic rectal cancer $(5,6)$. The high rates of local and distant relapse led to the standardization of optimal oncologic surgery in the form of the total mesorectal excision technique (7), as well as the adoption of adjuvant (chemo) radiotherapy in order to decrease the probability of local recurrence following the resection of high risk, T3-T4/N+ disease. Subsequently, neoadjuvant treatment was found to confer additional advantages in terms of patient outcomes and it was thus adopted as the standard of care (8-12). With various neoadjuvant chemoradiotherapy regimens used in recently reported trials, local control rates were largely less than $10 \%, 5$-year disease-free-survival rates ranged from $60-75 \%$, and pathologic complete response (pCR) rates ranged from $8-19.5 \%$ at the time of total mesorectal excision $(9,13,14)$.

This small, but not insignificant proportion of patients with no residual rectal adenocarcinoma at the time of surgery let some investigators to pursue an organpreservation approach with definitive chemoradiotherapy similar to what had been developed for squamous cell carcinoma of the anal canal (15-17). Investigators at Sao Paulo were the first to evaluate a selective non-operative strategy for patients with potentially resectable rectal adenocarcinoma who achieved a clinical complete response (cCR) to chemoradiotherapy (18-20). Subsequent groups sought to replicate the results of Habr-Gama et al and have achieved low local recurrence rates of $1-26 \%$, most of which are salvageable with surgery at recurrence (21-25).

Although a multi-institutional phase II study is currently underway (26), to date, the majority of published studies are largely small in size, single-institutional in nature and limited to a very well-selected patient population who achieve a cCR by various well-defined and stringent criteria. In practice, many patients who fall outside these selection criteria desire non-operative management for their localized rectal adenocarcinoma. Some patients may not be candidates for surgical intervention because of medical comorbidities, and some patients may refuse surgery even when it is recommended. There are currently limited data available to help counsel this population of patients as to their expected outcomes following a definitive radiotherapybased approach. Information in the National Cancer Database (NCDB) is particularly well-suited to address such a question given its large scale and varied treating institutional setting; and thus, it was used for this aim. In this study, we sought to describe the outcomes of patients in the NCDB treated with nonoperative management for localized rectal adenocarcinoma, evaluate any potential dose-response, and investigate other prognostic factors for overall survival (OS) in this population.

\section{Methods}

We first obtained the necessary ethical and regulatory institutional review board approval from the University of Texas MD Anderson Institutional Review Board as well as a waiver of informed consent as the information in the Commission on Cancer's NCDB is de-identified. Subsequently, we extracted the data of this analysis from the NCDB registry.

\section{Selection of the study cohort}

The initial cohort included 243,499 patients with rectal cancer (International Classification of Diseases for Oncology 3rd edition disease topography code C209) in the NCDB 2014 release, who were diagnosed from 2004-2014. We first restricted our analysis to the subset of patients who did not receive definitive surgery $(\mathrm{N}=57,583)$, and then we excluded patients who received no radiation $(\mathrm{N}=29,668)$, unknown radiation $(\mathrm{N}=1,081)$ or miscellaneous forms of radiation such as Gamma Knife, Linac-based radiosurgery or therapy with radioisotopes $(\mathrm{N}=109)$. This left us with a remaining cohort of 26,932 patients. Next, we limited our analysis to patients receiving radiation to the pelvic soft tissue $(\mathrm{N}=21,969)$ and excluded patients with stage 4 disease $(\mathrm{N}=6,215)$. We then excluded patients who received less than 45 Gray $(\mathrm{Gy})(\mathrm{N}=2,374)$ or had unknown/incomplete radiation records in the NCDB $(\mathrm{N}=958)$. This left us with a cohort of 12,044. Finally, we excluded patients who either did not have any follow up data available in the NCDB $(\mathrm{N}=1,512)$ or who died before surgery could be performed, 
for whom surgery was recommended but not performed or for whom the reason for omission of surgery was not provided $(\mathrm{N}=2,124)$. This left us with a final cohort of 8,408 patients for analysis. The patients in this final cohort had non-metastatic rectal adenocarcinoma treated with external beam radiation therapy to the pelvis a total dose of $45 \mathrm{~Gy}$ or higher who did not undergo a surgical resection either because the patient refused surgery or because surgery was medically contraindicated or otherwise unplanned.

\section{Data collection}

Data extracted from the NCDB for each case included patient-centric variables including age at diagnosis, sex (male or female) and race (White, Black, Asian/Pacific Islander, and other). Specific comorbidity information is not available in the NCDB, but a summary of the Charlson Comorbidity score $(0,1$ or $2+)$ was collected. Year of diagnosis was also recorded and subdivided into two groups ( $\leq 2009$ or $>2009$ ). Additionally, alpha-fetoprotein (AFP) level at diagnosis (in $\mathrm{ng} / \mathrm{mL}$ ) was obtained; although the exact value was not provided as a continuous variable, values were categorized in $\leq 19,20-99,100-499,500-999$ and $\geq 1,000 \mathrm{ng} / \mathrm{mL}$. As the data spanned the period from 2004-2014, patients diagnosed from 2004-2009 were staged using the 6th edition of the American Joint Committee on Cancer (AJCC) staging criteria and patients diagnosed from 2010-2014 were staged using the $7^{\text {th }}$ edition. Changes between the $6^{\text {th }}$ and $7^{\text {th }}$ editions included subdivision of T4 into T4a and T4b in the $7^{\text {th }}$ edition. However, we simplified all T-staging data in our analysis to T1, T2, T3 or T4 for consistency. Additionally, the $\mathrm{N}$-staging changed in the $7^{\text {th }}$ edition to subdivide N1 into N1a, N1b and N1c as well as to subdivide N2 into N2a and N2b. Again, for consistency, we simplified all $\mathrm{N}$-staging data in our analysis to $\mathrm{Nx}, \mathrm{N} 0$, $\mathrm{N} 1$ or N2.

Center-specific variables were also collected. Facility type was divided into community versus academic centers. Community center included facilities coded as either a community cancer program or a comprehensive community cancer program, while academic centers included facilities coded as either an academic/research program or an integrated network cancer program. Each patient's insurance status was recorded as none, Medicaid, Medicare, other government insurance or private insurance.

Finally, treatment-specific variables were recorded from the NCDB including the days from diagnosis to initiation of radiation therapy, radiation modality $3 \mathrm{D}$ conformal radiation therapy (3DCRT) or intensitymodulated radiation therapy (IMRT) and the total dose of radiation in Gy. Where applicable, the total dose was calculated as the initial dose followed by the boost dose. Total dose groups were subdivided into $<50.4,50.4-54$ and $>54$ Gy. Chemotherapy data were also collected including whether patients received no chemotherapy, single agent chemotherapy or combination chemotherapy either concurrently or sequentially with radiation. The reason definitive surgery was not performed was also recorded (patient refused $v s$. not recommended/contraindicated).

Median OS was calculated by examining months from diagnosis to last contact or death as well as the recorded vital status (dead or alive). Patients who were treated and then lost to follow up were censored at the date of their last recorded follow up.

\section{Statistical considerations}

We utilized descriptive statistics to describe baseline characteristics of included patients, Pearson Chi-square test to evaluate frequency of various characteristics according to dose level (<50.4, 50.4-54 and $>54$ Gy).

Kaplan-Meier survival estimates were used to evaluate OS, and log-rank testing was used for between-group survival comparisons. Kaplan-Meier survival curves were adjusted for significant variables as identified on the multivariate analysis.

Univariate and multivariate analyses were performed using Cox regression analysis to determine factors associated with improved OS; hazard ratios (HR) with corresponding $95 \%$ CI were accordingly generated. After a Bonferroni correction was applied accounting for the 13 variables tested in the univariate analysis, statistical significance was considered if a two-tailed $\mathrm{P}$ value less than 0.004 was achieved.

To adjust survival for the significant factors found on multivariable analysis, a robust inverse-probability-weighted regression adjustment (IPWRA) method was employed, and this analysis was augmented by the using of nearestneighbor matching. Nearest-neighbor matching estimators impute the missing potential outcome for each subject by using an average of the outcomes of similar subjects that receive the other treatment level. Similarity between subjects is based on a weighted function of the covariates for each observation. Treatment effects adjusted for in this analysis included sex, age, tumor size, Charlson-Deyo comorbidity score, N-stage, insurance status, chemotherapy 
type and radiation dose group (<50.4, 50.4-54 or $>54$ Gy). This IPWRA method was carried out with a nearest neighbor match of up to four cases, although a minimum of one match is required for this technique. All the statistical analyses were performed using Stata Statistical Software version 14 (College Station, TX, USA).

\section{Results}

\section{Baseline characteristics}

Eight thousand four hundred and eight patients with nonmetastatic rectal adenocarcinoma were included in the current study. Among which 2,122 patients (25.2\%) were treated with a total radiotherapy dose of 45-50.3 Gy, 4,707 patients $(56.0 \%)$ were treated with a total radiotherapy dose of 50.4-54 Gy, and 1,579 patients (18.8\%) were treated with a total radiotherapy dose $>54$ Gy. Table 1 summarizes baseline characteristics of included patients. Overall in the studied cohort, median age was 67 and there were more females than males (57.4\% vs. $42.6 \%)$; white race predominates $(82.4 \%)$ and most of the patients have a Charlson-Deyo comorbidity index of 0 (79.2\%). Most patients were treated at community centers $(58.7 \%)$ and almost half of the patients were covered with Medicare insurance $(52.7 \%)$. Median time from diagnosis to radiotherapy was 38 days (IQR: $23-54$ ) and most of the patients were treated with 3DCRT (84.1\%). Single agent concurrent (or sequential) chemotherapy was utilized in $52.8 \%$; while combination chemotherapy was utilized in $25.4 \%$. Patients did not receive surgical intervention because of patient refusal in $12.5 \%$ of the cases, because of medical contraindications in $9.7 \%$ of the cases and because of other reasons in $77.7 \%$ of the cases.

\section{Survival analyses}

Univariate and multivariate analyses were conducted to evaluate factors affecting OS in the study cohort (Table 2). Factors significantly associated with worse OS on multivariate analysis included male gender, age $>67$ years, Charlson-Deyo score $>0$, node-positive disease, having nonprivate medical insurance, not receiving chemotherapy and having medical contraindications to surgery as the reason of no surgical intervention. Patients who received moderate dose escalation (50.4-54 Gy) had significantly higher OS on multivariate analysis [HR 0.8 (95\% CI: 0.72-0.88); $\mathrm{P}<0.001]$ when compared with patients who received
45-50.3 Gy. Patients who received >54 Gy did not have a significantly different survival [HR 0.96 (95\% CI: 0.85-1.09); $\mathrm{P}=0.553]$ when compared with patients who received 45-50.3 Gy.

Figures 1,2 show Kaplan-Meier survival curves for the whole cohort of patients as well as for different dose subsets of the patients In Figure 2, treatment effects adjusted for in the IPWRA analysis included sex, age, tumor size, Charlson-Deyo comorbidity score, N-stage, insurance status, chemotherapy type and radiation dose group $(<50.4$, 50.4-54 or >54 Gy).

The median survival was 43.2 months for all included patients, with 1- and 5-year OS of 83.9\% (95\% CI: 83.1-84.7\%) and $41.3 \%$ (95\% CI: 40.1-42.5\%), respectively. After casematching and adjusting for other significant prognostic factors on IPWRA analysis (sex, age, tumor size, CharlsonDeyo comorbidity score, N-stage, insurance status, chemotherapy type and radiation dose group $(<50.4$, 50.4-54 or >54 Gy), patients receiving 50.4-54 Gy had a significantly longer median, 1 - and 5-year OS (49.4 months, $85.8 \%, 44.7 \%$ ) compared with patients receiving 45-50.3 or >54 Gy (37.2 months, 79.2\%, 38.4\% and 34.2 months, $84.5 \%, 35.3 \%$, respectively; Log rank P value $<0.0001$ ) (Table 3).

\section{Discussion}

The current study reports the outcomes of non-metastatic rectal adenocarcinoma patients treated with a radiationbased approach as a definitive treatment strategy in largescale, multi-institutional setting. It shows that in the unselected group of patients treated at NCDB-participating institutions (both academic and community-based), survival rates for patients treated with a non-surgical approach are much lower than those reported in previously reported studies with well-selected patient population. Interestingly in the current analysis, moderate dose escalation from 50.4-54 Gy seems to be associated with better survival outcomes compared with doses $<50.4$ or $>54$ Gy (after adjusting for other significant covariates).

The main strengths of the current analysis include the relatively large number of patients treated at a wide range of academic and community-based centers for a relatively uncommon clinical scenario. Moreover, compared to other available registry-based population studies (e.g., surveillance, epidemiology and end results-SEER database), the NCDB provides some additional details with regards to the comorbidity index as well as timing of different 
Table 1 Baseline characteristics of non-metastatic rectal adenocarcinoma patients with treated non-surgical management including radiation therapy to at least 45 Gy listed in the National Cancer Database between 2004 and 2014

\begin{tabular}{|c|c|c|c|c|c|}
\hline Characteristic & $\begin{array}{l}\text { All cases } \\
(\mathrm{N}=8,408)\end{array}$ & $\begin{array}{c}45-50.3 \text { Gy } \\
(\mathrm{N}=2,122,25.2 \%)\end{array}$ & $\begin{array}{c}50.4-54 \text { Gy } \\
(\mathrm{N}=4,707,56.0 \%)\end{array}$ & $\begin{array}{c}>54 \text { Gy } \\
(\mathrm{N}=1,579,18.8 \%)\end{array}$ & $P$ value and test \\
\hline Diagnosis year; N (\%) & & & & & $\begin{array}{l}<0.001 \text {; Pearson } \\
\text { Chi-square }\end{array}$ \\
\hline$\leq 2009$ & 4,496 (53.5) & $1,228(57.9)$ & $2,313(49.1)$ & $955(60.5)$ & \\
\hline$>2009$ & $3,912(46.5)$ & $894(42.1)$ & 2,394 (50.9) & $624(39.5)$ & \\
\hline Male & $3,580(42.6)$ & $930(43.8)$ & $1,871(39.7)$ & $779(49.3)$ & \\
\hline Female & $4,828(57.4)$ & 1,192 (56.2) & $2,836(60.3)$ & $800(50.7)$ & \\
\hline $\begin{array}{l}\text { Age in years; median } \\
\text { (IQR) }\end{array}$ & $67[56-78]$ & 68 [57-79] & $66[55-77]$ & 72 [60-84] & $\begin{array}{l}<0.001 \text {; nonparametric } \\
\text { equality of medians }\end{array}$ \\
\hline White & $6,931(82.4)$ & $1,708(80.5)$ & $3,860(82.0)$ & 1,363 (86.3) & \\
\hline Black & $1,011(12.0)$ & 287 (13.5) & $557(11.8)$ & $167(10.6)$ & \\
\hline Asian & 278 (3.3) & $70(3.3)$ & $181(3.8)$ & $27(1.7)$ & \\
\hline Other & $188(2.2)$ & $57(2.7)$ & $109(2.3)$ & $22(1.4)$ & \\
\hline $\begin{array}{l}\text { Charlson co-morbidity } \\
\text { score; N (\%) }\end{array}$ & & & & & $\begin{array}{l}<0.001 \text {; Pearson } \\
\text { Chi-square }\end{array}$ \\
\hline 0 & $6,660(79.2)$ & $1,678(79.1)$ & $3,777(80.2)$ & 1,205 (76.3) & \\
\hline 1 & $1,251(14.9)$ & $315(14.8)$ & $698(14.8)$ & $238(15.1)$ & \\
\hline $\mathrm{T} 2$ & $1,007(12.0)$ & $234(11.0)$ & $506(10.7)$ & 267 (16.9) & \\
\hline T3 & 4,356 (51.8) & $1,057(49.8)$ & $2,638(56.0)$ & $661(41.9)$ & \\
\hline $\mathrm{T} 4$ & $980(11.7)$ & $251(11.8)$ & $522(11.1)$ & $207(13.1)$ & \\
\hline Tx/missing & $1,472(17.5)$ & 409 (19.3) & 717 (15.2) & $302(19.1)$ & \\
\hline N stage ${ }^{1} ; \mathrm{N}(\%)$ & & & & & $\begin{array}{l}<0.001 \text {; Pearson } \\
\text { Chi-square }\end{array}$ \\
\hline No & 4,438 (52.8) & $1,097(51.7)$ & 2,455 (52.2) & $886(56.1)$ & \\
\hline N1 & $2,273(27.0)$ & $555(26.2)$ & $1,384(29.4)$ & 334 (21.1) & \\
\hline N2 & $420(5.0)$ & $104(4.9)$ & $233(5.0)$ & $83(5.3)$ & \\
\hline $\mathrm{Nx}$ & 1,277 (15.2) & 366 (17.2) & 635 (13.5) & $276(17.5)$ & \\
\hline
\end{tabular}

Table 1 (continued) 
Table 1 (continued)

\begin{tabular}{|c|c|c|c|c|c|}
\hline Characteristic & $\begin{array}{l}\text { All cases } \\
(\mathrm{N}=8,408)\end{array}$ & $\begin{array}{c}45-50.3 \text { Gy } \\
(\mathrm{N}=2,122,25.2 \%)\end{array}$ & $\begin{array}{c}50.4-54 \text { Gy } \\
(\mathrm{N}=4,707,56.0 \%)\end{array}$ & $\begin{array}{c}>54 \text { Gy } \\
(\mathrm{N}=1,579,18.8 \%)\end{array}$ & $P$ value and test \\
\hline Facility type ${ }^{2} ; \mathrm{N}(\%)$ & & & & & $\begin{array}{l}<0.001 \text {; Pearson } \\
\text { Chi-square }\end{array}$ \\
\hline Community center & 4,936 (58.7) & $1,276(60.1)$ & $2,674(56.8)$ & $986(62.4)$ & \\
\hline Academic center & 3,247 (38.6) & 784 (36.9) & $1,898(40.3)$ & $565(35.8)$ & \\
\hline Insurance; N (\%) & & & & & $\begin{array}{l}<0.001 \text {; Pearson Chi- } \\
\text { square }\end{array}$ \\
\hline Uninsured & 437 (5.2) & $113(5.3)$ & $257(5.5)$ & $67(4.2)$ & \\
\hline Private & 2,594 (30.9) & $643(30.3)$ & 1,561 (33.2) & $390(24.7)$ & \\
\hline Other/Govt & $212(2.5)$ & $43(2.0)$ & $135(2.9)$ & $34(2.2)$ & \\
\hline Unknown & $145(1.7)$ & $41(1.9)$ & $79(1.7)$ & $25(1.6)$ & \\
\hline $\begin{array}{l}\text { Diagnosis to } \\
\text { radiotherapy in days; } \\
\text { median (IQR) }\end{array}$ & 38 [23-54] & 38 [22-55] & 38 [23-53] & 35 [20-51] & $\begin{array}{l}0.027 \text {; nonparametric } \\
\text { equality of medians }\end{array}$ \\
\hline $\begin{array}{l}\text { Radiotherapy } \\
\text { modality; N (\%) }\end{array}$ & & & & & $\begin{array}{l}<0.001 \text {; Pearson } \\
\text { Chi-square }\end{array}$ \\
\hline IMRT & $1,337(15.9)$ & $327(15.4)$ & $688(14.6)$ & $322(20.4)$ & \\
\hline 3DCRT & $7,071(84.1)$ & $1,795(84.6)$ & $4,019(85.4)$ & $1,257(79.6)$ & \\
\hline Single agent & $4,441(52.8)$ & $1,137(5.4)$ & $2,623(55.7)$ & $681(43.1)$ & \\
\hline Combination & $2,133(25.4)$ & $481(22.7)$ & $1,154(24.5)$ & $498(31.5)$ & \\
\hline Unknown & $763(9.1)$ & $209(9.8)$ & $431(9.2)$ & $123(7.8)$ & \\
\hline $\begin{array}{l}\text { Reason for no surgery; } \\
\mathrm{N}(\%)\end{array}$ & & & & & $\begin{array}{l}<0.001 \text {; Pearson } \\
\text { Chi-square }\end{array}$ \\
\hline Patient refused & $1,054(12.5)$ & $241(11.4)$ & $602(12.8)$ & $211(13.4)$ & \\
\hline $\begin{array}{l}\text { Medically } \\
\text { contraindicated }\end{array}$ & $819(9.7)$ & $182(8.6)$ & $409(8.7)$ & $288(14.4)$ & \\
\hline $\begin{array}{l}\text { Otherwise not } \\
\text { planned }\end{array}$ & 6,535 (77.7) & $1,699(80.1)$ & 3,696 (78.5) & $1,140(72.2)$ & \\
\hline
\end{tabular}

Gy, Gray; IQR, interquartile range; IMRT, intensity-modulated radiation therapy; 3DCRT, 3D conformal radiation therapy; Govt, government. 1 , patients diagnosed 2004-2009 were staged according to the $6^{\text {th }}$ edition of the TNM system whereas patients diagnosed $2010-2014$ were staged according to the $7^{\text {th }}$ edition. Patients with distant metastatic disease were excluded; ${ }^{2}$, community centers include: Ccommunity Cancer Programs and Comprehensive Community Cancer Programs; whereas academic centers include Academic/Research Programs and Integrated Network Cancer Programs. 
Table 2 Univariate and multivariate analysis for factors affecting overall survival in the entire cohort

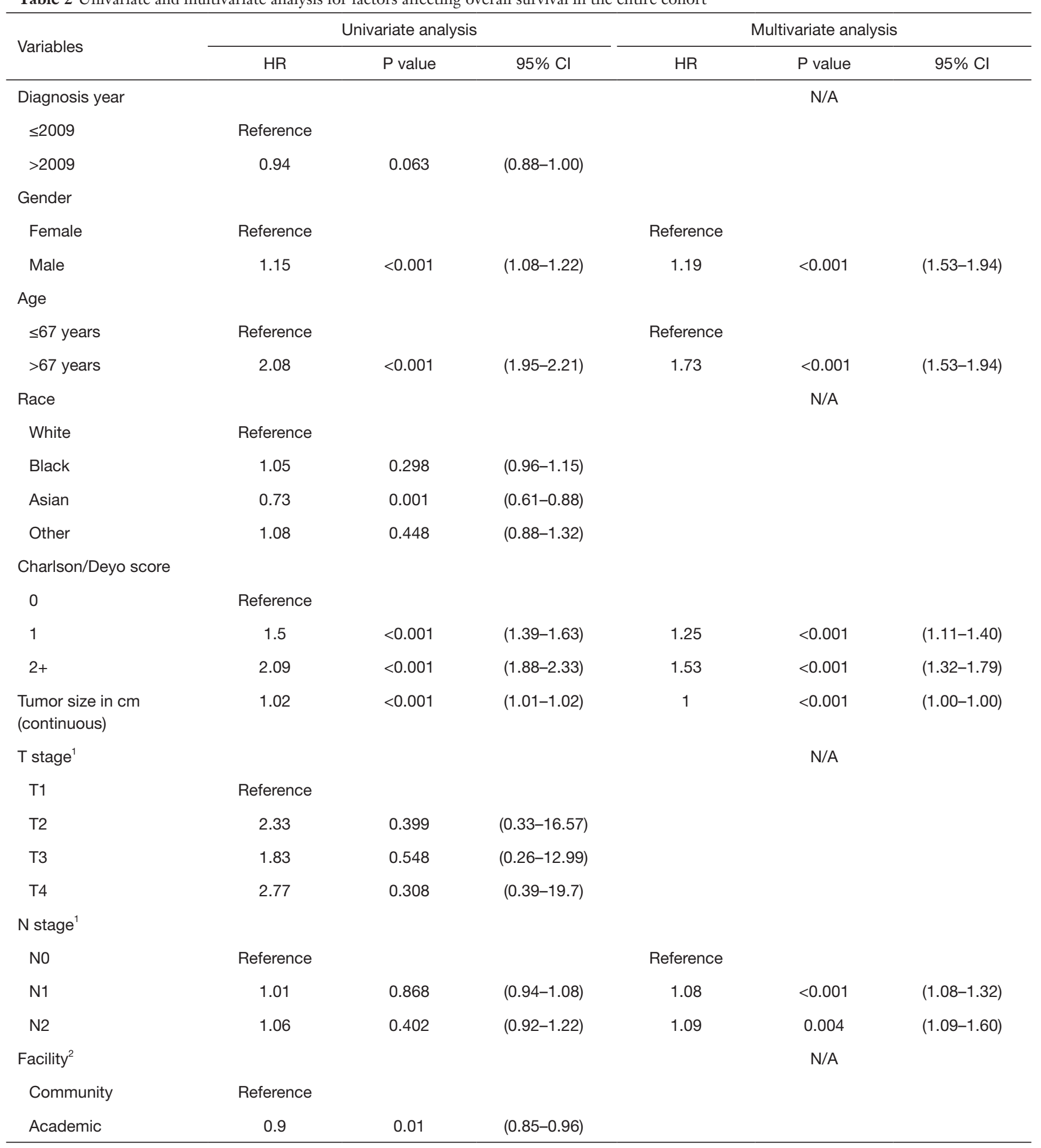

Table 2 (continued) 
Table 2 (continued)

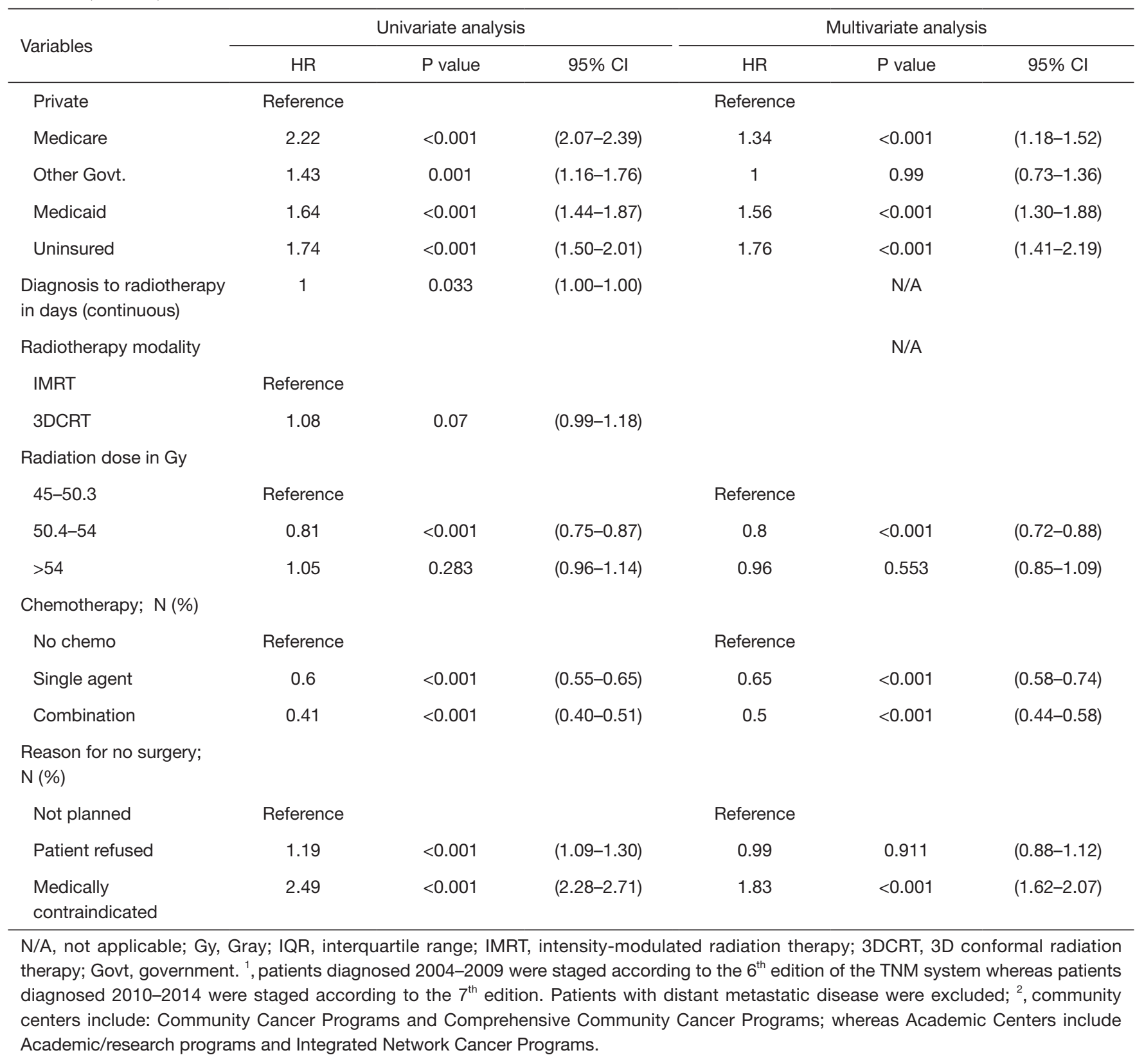

treatments (including chemotherapy and radiotherapy).

However, there are also several important limitations to bear in mind when interpreting data from the NCDB. There is the potential for miscoding which can add statistical noise. There is also an undeniable selection bias in clinical practice that can be difficult to tease out, even after controlling for other factors. The CharlsonDeyo score is helpful, but the absence of more detailed information about performance status of the patients is problematic, particularly when looking at a population of patients who were not offered surgery or who refused surgery. Additionally, the NCDB does not include pertinent treatment information such as specific agents/doses/ schedules of chemotherapy. Moreover, and like other retrospective registry-based studies, the current study is subject to confounding related to the uncontrolled nature of primary data collection. It has also to be noted that the neither the NCDB, nor most of the other large-scale cancer 


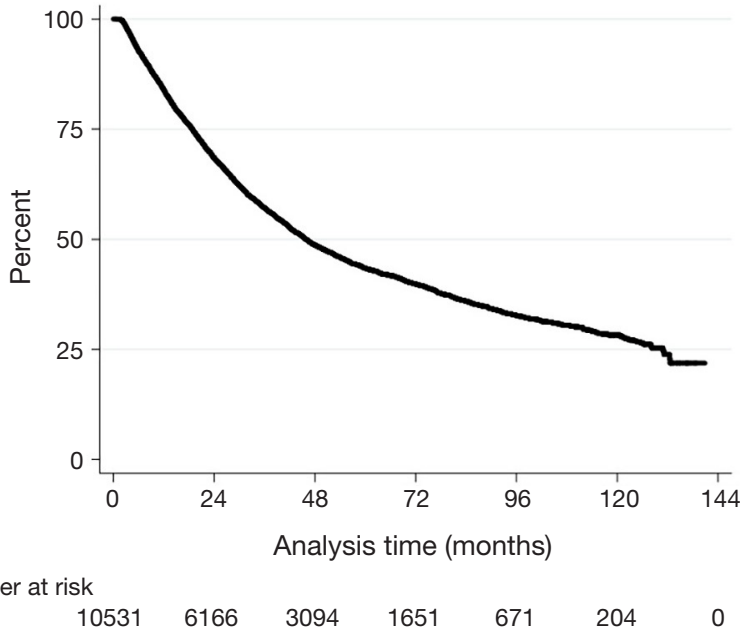

Figure 1 Overall survival for the entire cohort. registries (e.g., SEER) do not provide information about the extent of clinical response post chemoradiation. This piece of information particularly was shown to predict the outcomes of anal and rectal cancer patients treated with upfront chemoradiation. Thus, the main utility of the results of the current analysis may be in counseling patients who either decline surgery or who are not medically candidates for surgery about their expected outcomes with a completely non-surgical approach.

In general, the OS outcomes reported in this study in this population of patients treated non-surgically is quite low. Based on the Charlson-Deyo score reported in the NCDB, $79 \%$ of the study population had a comorbidity score of 0 , and only approximately $10 \%$ of patients had medical contraindications to surgical intervention. This

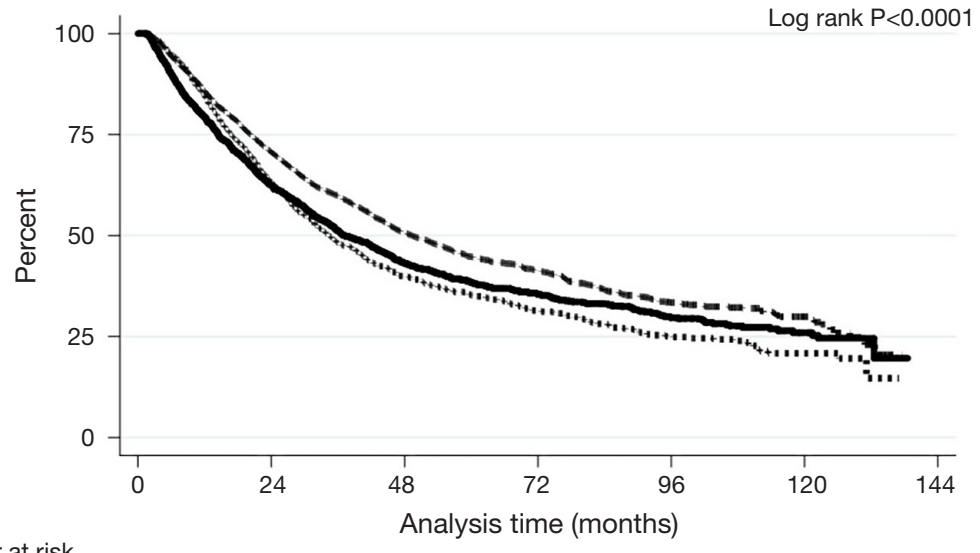

\begin{tabular}{|c|c|c|c|c|c|c|c|c|}
\hline \multicolumn{9}{|c|}{ Number at risk } \\
\hline & $<504$ GV & 2121 & & & & 110 & 17 & 0 \\
\hline & 50.4-54 Gy & 4707 & 2800 & 1341 & 672 & 257 & 78 & 0 \\
\hline ..... & $>54 \mathrm{~Gy}$ & 1579 & 874 & 406 & 231 & 100 & 22 & 0 \\
\hline
\end{tabular}

Figure 2 Overall survival for matched cases by dose group.

Table 3 Overall survivals for matched cases utilizing an inverse-probability weight regression adjustment using nearest neighbor matching

\begin{tabular}{|c|c|c|c|c|}
\hline Outcomes & $45-50.3$ Gy $(N=2,121)$ & $50.4-54$ Gy $(N=4,707)$ & >54 Gy $(\mathrm{N}=1,579)$ & Log rank $P$ value \\
\hline Mean OS in months $(95 \% \mathrm{Cl})$ & $77.7(67.6-87.7)$ & $82.2(76.1-88.4)$ & $72.7(63.6-81.9)$ & $<0.0001$ \\
\hline Median OS in months & 37.2 & 49.4 & 34.2 & \\
\hline 2-year OS (95\% Cl) (\%) & $62.4 \%(60.3-64.5)$ & $70.7 \%(69.3-72.0)$ & $63.4 \%(60.9-65.8)$ & \\
\hline 3-year OS (95\% Cl) (\%) & $51.2 \%(48.9-53.4)$ & $59.8 \%(58.3-61.3)$ & $48.2 \%(45.5-50.8)$ & \\
\hline 5-year OS (95\% Cl) (\%) & $38.4 \%(36.0-40.8)$ & $44.7 \%(43.0-46.4)$ & $35.3 \%(32.6-38.0)$ & \\
\hline 6 -year OS (95\% Cl) (\%) & $35.5 \%(33.1-38.0)$ & $41.3 \%(39.5-43.1)$ & $31.4 \%(28.7-34.2)$ & \\
\hline 12 -year OS (95\% Cl) (\%) & $25.9 \%(22.9-29.1)$ & $29.9 \%(27.4-32.4)$ & $20.9 \%(17.6-24.3)$ & \\
\hline
\end{tabular}

Gy, Gray; OS, overall survival; Cl, confidence interval. 
would suggest that the study population is relatively healthy at baseline, so the question becomes: what is the cause of the high mortality? T-stage is not prognostic for OS in our multivariate analysis for this population; however, we know from a plethora of other studies that stage is of enormous prognostic value for rectal cancer. In our analysis, age and Charlson-Deyo comorbidity index were the primary drivers of OS. This raises the question of whether the patients in this cohort might be dying from non-cancer related causes. The fact that the NCDB provides no data on cancer-specific survival, unfortunately, leaves this question unresolved.

We restricted the current analysis to patients who received long-course, standard fractionation (chemo)radiotherapy. The rationale for this approach is the fact that long-course preoperative radiotherapy was associated with a higher probability of pathological complete response compared to short-course preoperative radiotherapy in previous publications $(12,13)$. Although specific chemotherapy details are lacking in the NCDB, almost half of all cases received single agent concurrent (+/- sequential) chemotherapy together with long course radiation therapy; while only $25 \%$ of patients received combination chemotherapy. Previous studies failed to show a benefit to combination chemotherapy (e.g., oxaliplatin/fluoropyrimidine) compared to fluoropyrimidine alone in the neoadjuvant treatment of rectal cancer $(14,15)$. However, it remains to be seen if combination chemotherapy may add to the management of non-surgically treated patients.

Moreover, it is customary to administer chemotherapy concurrent with radiotherapy in the neoadjuvant treatment of rectal cancer $(16,17)$. Whether other treatment schedules (e.g., induction chemotherapy followed by concurrent chemoradiotherapy or concurrent chemoradiotherapy followed by consolidation chemotherapy) might be of additional help to non-surgically treated patients is not yet known with certainty. Moreover, the additional role of targeted treatment is unclear in the setting of the nonsurgically treated patients.

The majority of patients in the current study were treated with 3DCRT and the dose varied from 45 to $>54$ Gy. Previous studies have shown non-conclusive results about the benefit from boost in the neoadjuvant setting (particularly given the excess acute toxicities associated with increased doses) (18-20). The current study suggested also that doses above 54 Gy might actually be detrimental. However, the fact that toxicity data are not available in the NCDB does not allow us to evaluate further why that might be the case. It stands to reason that moderate dose escalation may improve cancer control which may, in turn, lead to improved OS. However, given the inherent uncertainty of the registry-based retrospective analyses, these results need to be confirmed prospectively.

Recent years have witnessed impressive advances in the radiotherapy planning strategies of rectal cancer in the neoadjuvant setting. Incorporation of functional imaging techniques (e.g., PET/CT scan) is particularly attractive in this setting with encouraging results from recent dosimetric studies (21). The impact of these advances on the planning of non-surgically treated patients is not yet clear. In conclusion, in the unselected group of patients treated at NCDB-participating institutions, survival rates with a nonsurgical approach are much lower than those reported in previously reported studies with well-selected patient population. Moderate dose escalation from 50.4-54 Gy seems to be associated with better survival outcomes compared with doses $<50.4$ or $>54 \mathrm{~Gy}$.

\section{Acknowledgements}

None.

\section{Footnote}

Conflicts of Interest: The authors have no conflicts of interest to declare.

Ethical Statement: We first obtained the necessary ethical and regulatory institutional review board approval from the University of Texas MD Anderson Institutional Review Board (No. PA17-0278) as well as a waiver of informed consent as the information in the Commission on Cancer's NCDB is de-identified.

\section{References}

1. Siegel RL, Miller KD, Fedewa SA, et al. Colorectal cancer statistics, 2017. CA Cancer J Clin 2017;67:177-93.

2. Nelson H, Petrelli N, Carlin A, et al. Guidelines 2000 for colon and rectal cancer surgery. J Natl Cancer Inst 2001;93:583-96.

3. Wiltink LM, Marijnen CA, Meershoek-Klein Kranenbarg E, et al. A comprehensive longitudinal overview of health-related quality of life and symptoms after treatment for rectal cancer in the TME trial. Acta 
Oncol 2016;5 5:502-8.

4. Adjuvant therapy for patients with colon and rectal cancer: summary of NIH consensus statement. National Cancer Institute and the Office of Medical Applications of Research of the National Institutes of Health. Aust N Z J Surg 1991;61:23.

5. Gill PG, Ludbrook J. Cancer: an indictment against radical surgery. Med J Aust 1975;2:226-8.

6. Wong RKS, Tandan V, De Silva S, et al. Pre-operative radiotherapy and curative surgery for the management of localized rectal carcinoma. Cochrane Database Syst Rev 2007;(2):CD002102.

7. Enker WE. Total mesorectal excision--the new golden standard of surgery for rectal cancer. Ann Med 1997;29:127-33.

8. Kennedy E, Vella ET, Blair Macdonald D, et al. Optimisation of preoperative assessment in patients diagnosed with rectal cancer. Clin Oncol R Coll Radiol 2015;27:225-45.

9. Sauer R, Liersch T, Merkel S, et al. Preoperative versus postoperative chemoradiotherapy for locally advanced rectal cancer: results of the German CAO/ARO/AIO-94 randomized phase III trial after a median follow-up of 11 years. J Clin Oncol 2012;30:1926-33.

10. Roh MS, Colangelo LH, O'Connell MJ, et al. Preoperative multimodality therapy improves disease-free survival in patients with carcinoma of the rectum: NSABP R-03. J Clin Oncol 2009;27:5124-30.

11. Park JH, Yoon SM, Yu CS, et al. Randomized phase 3 trial comparing preoperative and postoperative chemoradiotherapy with capecitabine for locally advanced rectal cancer. Cancer 2011;117:3703-12.

12. NCCN. National Comprehensive Cancer Network Guidelines Version 3.2017 Rectal Cancer. Available online: https://www.nccn.org/professionals/physician_gls/pdf/ rectal.pdf

13. Gérard JP, Azria D, Gourgou-Bourgade S, et al. Comparison of two neoadjuvant chemoradiotherapy regimens for locally advanced rectal cancer: results of the phase III trial ACCORD 12/0405-Prodige 2. J Clin Oncol 2010;28:1638-44.

14. O'Connell MJ, Colangelo LH, Beart RW, et al. Capecitabine and oxaliplatin in the preoperative multimodality treatment of rectal cancer: surgical end points from National Surgical Adjuvant Breast and Bowel Project trial R-04. J Clin Oncol 2014;32:1927-34.

15. Rich TA, Ajani JA, Morrison WH, et al. Chemoradiation therapy for anal cancer: radiation plus continuous infusion of 5 -fluorouracil with or without cisplatin. Radiother Oncol 1993;27:209-15.

16. Ajani JA, Winter KA, Gunderson LL, et al. Fluorouracil, mitomycin, and radiotherapy vs fluorouracil, cisplatin, and radiotherapy for carcinoma of the anal canal: a randomized controlled trial. JAMA 2008;299:1914-21.

17. Kachnic LA, Winter K, Myerson RJ, et al. RTOG 0529: a phase 2 evaluation of dose-painted intensity modulated radiation therapy in combination with 5 -fluorouracil and mitomycin-C for the reduction of acute morbidity in carcinoma of the anal canal. Int J Radiat Oncol Biol Phys 2013;86:27-33.

18. Habr-Gama A, Perez RO, Nadalin W, et al. Operative versus nonoperative treatment for stage 0 distal rectal cancer following chemoradiation therapy: long-term results. Ann Surg 2004;240:711-7; discussion 717-8.

19. Habr-Gama A, Perez RO. Non-operative management of rectal cancer after neoadjuvant chemoradiation. Br J Surg 2009;96:125-7.

20. Habr-Gama A, Sabbaga J, Gama-Rodrigues J, et al. Watch and wait approach following extended neoadjuvant chemoradiation for distal rectal cancer: are we getting closer to anal cancer management? Dis Colon Rectum 2013;56:1109-17.

21. Maas M, Beets-Tan RG, Lambregts DM, et al. Waitand-see policy for clinical complete responders after chemoradiation for rectal cancer. J Clin Oncol 2011;29:4633-40.

22. Martens MH, Maas M, Heijnen LA, et al. Long-term Outcome of an Organ Preservation Program After Neoadjuvant Treatment for Rectal Cancer. J Natl Cancer Inst 2016;108.

23. Appelt AL, Pløen J, Harling H, et al. High-dose chemoradiotherapy and watchful waiting for distal rectal cancer: a prospective observational study. Lancet Oncol 2015;16:919-27.

24. Beets GL, Figueiredo NL, Habr-Gama A, et al. A new paradigm for rectal cancer: Organ preservation: Introducing the International Watch \& Wait Database (IWWD). Eur J Surg Oncol 2015;41:1562-64.

25. Renehan AG, Malcomson L, Emsley R, et al. Watchand-wait approach versus surgical resection after chemoradiotherapy for patients with rectal cancer (the OnCoRe project): a propensity-score matched cohort analysis. Lancet Oncol 2016;17:174-83.

26. Smith JJ, Chow OS, Gollub MJ, et al. Organ Preservation in Rectal Adenocarcinoma: a phase II 
randomized controlled trial evaluating 3-year diseasefree survival in patients with locally advanced rectal cancer treated with chemoradiation plus induction or consolidation chemotherapy, and total mesorectal excision or nonoperative management. BMC Cancer 2015;15:767.

Cite this article as: Holliday EB, Allen PK, Elhalawani H, Abdel-Rahman O. Outcomes of patients in the national cancer database treated non-surgically for localized rectal cancer. J Gastrointest Oncol 2018;9(4):589-600. doi: 10.21037/ jgo.2018.03.06 